\title{
Bioinformatics analysis on the differentiation of bone mesenchymal stem cells into osteoblasts and adipocytes
}

\author{
XIAOYUAN XU, HE JIANG, XINGNUAN LI, PING WU, JIANYUN LIU, \\ TAO WANG, XIAOOU ZHOU, JIANJUN XIONG and WEIDONG LI
}

Key Laboratory of System Bio-Medicine of Jiangxi Province, Jiujiang University, Jiujiang, Jiangxi 332000, P.R. China

Received November 25, 2015; Accepted December 15, 2016

DOI: $10.3892 / \mathrm{mmr} .2017 .6178$

\begin{abstract}
The present study aimed to screen several differentially expressed genes (DEGs) and differentially expressed microRNAs (miRNAs) for two types of mesenchymal stem cell (MSC) differentiation. Bone morphogenetic protein 6 (BMP-6) and dexamethasone were used to induce MSCs towards osteoblastic differentiation or adipocytic differentiation. The $t$-test in the Bioconductor bioinformatics software tool was used to screen DEGs and differentially expressed miRNAs in the two samples. Subsequent gene ontology (GO) and pathway analyses on the DEGs were performed using the GO and Kyoto Encyclopedia of Genes and Genomes databases, respectively; potential target genes for the screened miRNAs were predicted using the TargetScan database. In addition, an interaction network between the DEGs and miRNAs was constructed. Numerous DEGs and miRNAs were screened during osteoblastic and adipocytic differentiation of MSCs. Important pathways, such as glutathione metabolism, pathogenic Escherichia coli infection and Parkinson's disease, and GO terms, including cytoskeletal protein binding and phospholipase inhibitor activity, were enriched in the screened DEGs from MSCs undergoing osteogenic differentiation and adipocytic differentiation. miRNAs, including miRNA (miR)-382 and miR-203, and DEGs, including neuronal growth regulator 1 (NEGR1), phosphatidic acid phosphatase 2B (PPAP2B), platelet-derived growth factor receptor alpha (PDGFRA), interleukin 6 signal transducer (IL6ST) and sortilin 1 (SORT1), were demonstrated to be involved in osteoblastic differentiation. In addition, the downregulated miRNAs (including miR-495, miR-376a and miR-543), the upregulated miR-106a, the upregulated DEGs, including enabled homolog (ENAH), polypeptide $N$-acetylgalactosaminyltransferase
\end{abstract}

Correspondence to: Dr Weidong Li, Key Laboratory of System Bio-Medicine of Jiangxi Province, Jiujiang University, 17 Lufeng Road, Jiujiang, Jiangxi 332000, P.R. China

E-mail: liweidong@outlook.com; liweidong55880@126.com

Key words: bone mesenchymal stem cells, osteoblast, adipocyte, differentiation, bioinformatics analysis
1 and acyl-CoA synthetase long-chain family member 1 , and the downregulated repulsive guidance molecule family member B and semaphorin SEMA7A were demonstrated to be involved in adipocytic differentiation. The results of the present study suggested that miRNAs (miR-203 and miR-382) and DEGs (NEGR1, PPAP2B, PDGFRA, IL6ST and SORT1) may serve pivotal functions in the osteoblastic differentiation of MSCs, whereas miR-495, which is also involved in osteoblast differentiation and had four targets, including NEGR1, miR-376a, miR-543 and ENAH may have crucial roles in adipocytic differentiation of MSCs.

\section{Introduction}

Mesenchymal stem cells (MSCs) were discovered in bone marrow and are multipotent stromal cells that have the ability to differentiate into numerous cell types (1). Therefore, MSCs have become one of the most promising seed cells for the treatment of osteoblastic or cardiovascular diseases $(2,3)$. Osteoblastic or adipocytic differentiation occurs when MSCs are cultured in three conditions in vitro: i) Three-dimensional culture format; ii) serum-free nutrient medium; and iii) with the addition of inducible factors, such as bone morphogenetic protein 6 (BMP-6) and dexamethasone (4). BMP-6 or dexamethasone may induce osteoblastic or adipocytic differentiation of MSCs, respectively, via crosstalk with signaling pathways, including mitogen-activated protein kinase, Wnt, Notch and nuclear factor-kappa B (5-7).

Osteoblast- and adipocyte-associated diseases, such as bone disorders, cartilage injury, bone damage, angiopathy and liposarcoma, put tremendous burden on patients worldwide (8-10). Previous studies have demonstrated that although certain treatments have been successful, overall treatment outcomes remain unsatisfactory $(11,12)$. Various studies have examined the differential expression of genes or microRNAs (miRNAs) during MSC differentiation using bioinformatics. For example, Chen et al (13) demonstrated that MSCs secreted microparticles enriched in pre-miRNAs. De Luca et al (14) revealed that epidermal growth factor receptor signaling affected the secretome of MSCs in breast cancer. Mrugala et al (15) performed large-scale expression profiling using DNA microarrays on MSCs at different time points during chondrogenic differentiation. Although a number of studies have screened for differentially expressed genes (DEGs) or differentially 
expressed miRNAs during MSC differentiation into osteoblasts or adipocytes, an association between these two differentiation pathways has rarely been examined.

The present study used RNA sequencing (RNA-seq) analysis to screen for DEGs and differentially expressed miRNAs during the differentiation of MSCs into osteoblasts or adipocytes. Comprehensive bioinformatics methods were used to analyze the functions of DEGs and to investigate the interaction between DEGs and differentially expressed miRNAs. The present study aimed to screen and identify target genes and miRNAs that are different between osteoblastic and adipocytic differentiation of MSCs, which may provide a theoretical basis for targeted prevention in MSC directional differentiation.

\section{Materials and methods}

MSC differentiation induction. Human MSCs were obtained from Biomedical Sciences Cell Center of Fudan University (Shanghai, China) and cultured in Dulbecco's modified Eagle's medium-low glucose (DMEM-LG; Gibco; Thermo Fisher Scientific, Inc., Waltham, MA, USA) containing 10\% fetal bovine serum (Hyclone; GE Healthcare, Logan, UT, USA), $100 \mathrm{U} / \mathrm{ml}$ penicillin and $100 \mathrm{U} / \mathrm{ml}$ streptomycin (Gibco; Thermo Fisher Scientific, Inc.) in an incubator at $37^{\circ} \mathrm{C}$ with $5 \% \mathrm{CO}_{2}$. Medium was changed every other day; non-adherent cells were removed and MSCs were cultured to passage 3.

Passage 3 MSCs $\left(5 \times 10^{4}\right.$ per well) were seeded on 6 -well plates and cultured in DMEM-LG and induced towards osteoblastic differentiation by adding $1 \times 10^{-9} \mathrm{~mol} / \mathrm{l}$ dexamethasone (Sigma-Aldrich; Merck Millipore, Darmstadt, Germany), $10 \mathrm{mmol} / \mathrm{l} \beta$-glycerophosphoric acid, disodium salt pentahydrate (Meryer Chemical Technology Co., Ltd., Shanghai, China) and $0.2 \mathrm{mmol} / 1$ sodium ascorbate (Merck Millipore). Adipocytic differentiation was induced by culturing MSCs in DMEM-high glucose medium with $1 \times 10^{-7} \mathrm{~mol} / \mathrm{l}$ dexamethasone, $0.5 \mathrm{mmol} / 1 \mathrm{3}$-isobutyl-1-methylxanthine and $0.05 \mathrm{mmol} / 1$ indomethacin. Prior to induction, $50 \mathrm{ng} / \mathrm{ml}$ BMP-6 (Prospec-Tany TechnoGene Ltd., East Brunswick, NJ, USA) was added to each well and cells were cultured for $24 \mathrm{~h}$. The induced samples were separated into 3 groups based on the time point (n=3/group): i) Osteoblast (OB)/at day (AD) 7, the BMP-6 induced sample at 7 days; ii) OB/AD14, the BMP-6 induced sample at 14 days; and iii) OB/AD21, the BMP-6 induced sample at 21 days. Induced MSCs without BMP-6 at 0 day were used as the control $(n=3)$.

Data preprocessing. Total RNAs was extracted using TRIzol reagent (Invitrogen; Thermo Fisher Scientific, Inc.) and the quality of RNAs were evaluated by modified formaldehyde agarose gel electrophoresis and $\mathrm{OD}_{260} / \mathrm{OD}_{280}$ absorbance ratio detection using a Genova Nano spectrophotometer (Bibby Scientific; Cole-Palmer, Stone, UK). RNA-seq data from the osteoblastic or adipocytic differentiation induced MSCs were obtained at 7, 14 or 21 days using SMARTer Universal Low Input RNA kit for sequencing (Clontech Laboratories, Inc., Mountainview, CA, USA), according to the manufacturer's protocol. The Fast-QC software (Babraham Bioinformatics, Cambridge, UK; http://www.bioinformatics.babraham. ac.uk/projects/fastqc) was used to assess the data, including the quality value distribution of bases. RNA sequences were subsequently mapped to human genome sequences using TopHat software 1.3 release (Center for Computational Biology, John Hopkins University, Baltimore, MD, USA; https://ccb.jhu.edu/software/tophat/index.shtml) (16). Expression values of mRNAs were calculated based on the fragments per kilobase of exon, per million of fragments mapped and fragment length.

Screening differentially expressed $m R N A$ and miRNA. DEGs in each group at each of the three time points were screened and compared with the control (cells at day 0 that had not been exposed to BMP-6) using the $t$-test function in Bioconductor 3.4 (https://bioconductor.org) with a false discovery rate $<0.05$ and $\log _{2} \mathrm{FCl}>2$, where FC is fold change. In addition, differentially expressed miRNAs at each time point were screened compared with the control using $\log _{2} \mathrm{FCl}>2$.

Series cluster analysis. The DEGs and differentially expressed miRNAs screened from four time point samples ( $0,7,14$ and 21 days) were used for series cluster analysis. The correlation between time and expressed value of differentially expressed genes/miRNAs was calculated using the agglomerative hierarchical clustering method using SPSS 19.0 (IBM SPSS, Armonk, NY, USA); the correlation index was 0.85 for series cluster analysis.

Gene ontology (GO) and Kyoto Encyclopedia of Genes and Genomes (KEGG) pathway enrichment analysis of DEGs. To further explore the functions of selected DEGs, biological processes and pathways were analyzed using the GO database (http://www.geneontology.org/page/go-database) and the $K E G G$ database (http://www.genome.jp/kegg), respectively $(17,18)$. Enrichment analysis was performed by Fisher's exact test using the Database for Annotation, Visualization and Integrated Discovery (https://david.ncifcrf.gov) bioinformatics resource based on a hypergeometric distribution (19); $\mathrm{P}<0.01$ was used as the threshold.

Target gene prediction for differentially expressed miRNAs. All screened differentially expressed miRNAs were imported into the TargetScanHuman database version 7.1 (http://www. targetscan.org/vert_71) to predict miRNA target genes (20).

Construction of miRNA-mRNA interaction network. Since osteoblastic and adipocytic differentiation were both induced from the MSCs, the DEGs with opposite expression characteristics were used to indicate the differentiation status of the two types of cells. To investigate the negative correlations between the DEGs and differentially expressed miRNAs, a miRNA-mRNA interaction network was constructed with the protein-protein interaction network using Cytoscape 3.4.0 software (http://www.cytoscape.org) and the Reactome Pathway Database (http://www.reactome.org); miRNAs and mRNAs with a plus tendency value of 25 were chosen for interaction pairs.

\section{Results}

Screening differentiallyexpressedmRNAs andmiRNAs.DEGs that were identified in the BMP-6/dexamethasone-induced 


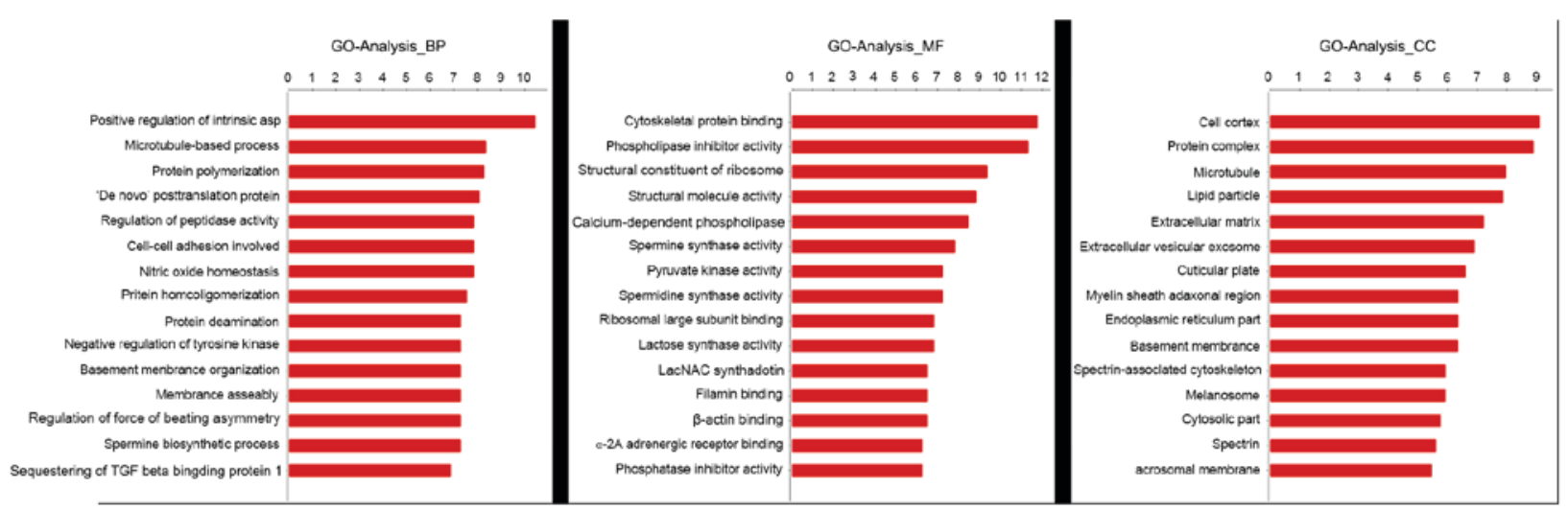

Figure 1. Enriched significant gene ontology analysis for the differentially expressed genes during mesenchymal stem cell differentiation. BP, biological process; CC, cellular component; MF, molecular function.

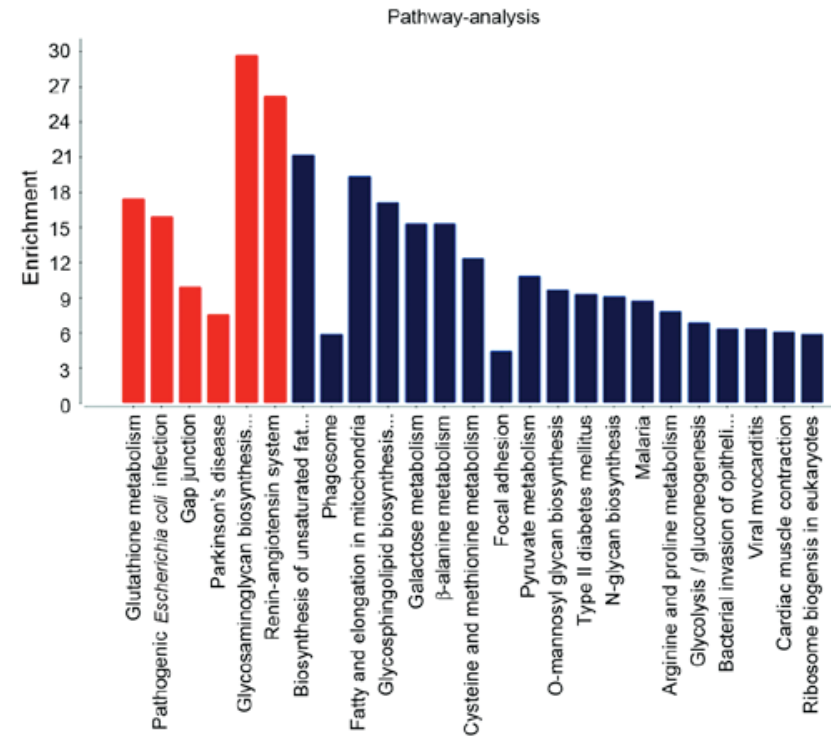

Figure 2. Kyoto Encyclopedia of Genes and Genomes enriched pathway analysis for the differentially expressed genes during differentiation of mesenchymal stem cells. Enrichment analysis was performed by Fisher's exact test. $\mathrm{P}<0.01$ was used as the threshold. Red color indicated significance and black non-significance.

samples at three time points (7, 14 and 21 days) were screened and compared with the control samples (day 0) that were not treated with BMP-6. Among the cells undergoing osteoblastic differentiation, 148 (58 upregulated and 90 downregulated) DEGs were selected at the 7-day time point, 293 (181 upregulated and 112 downregulated) at day 14 and 417 (253 upregulated and 164 downregulated) at day 21; whereas 45 (33 upregulated and 12 downregulated), 28 (11 upregulated and 17 downregulated) and 203 (21 upregulated and 182 downregulated) differentially expressed miRNAs were selected at the respective time points. Among the adipocyte differentiating groups, 572 (340 upregulated and 232 downregulated) DEGs were selected at the 7-day time point, 753 (419 upregulated and 334 downregulated) at day 14 and 829 (466 upregulated and 363 downregulated) at day 21. A total of 48 (20 upregulated and 28 downregulated), 48 (22 upregulated and 26 downregulated) and 66 (26 upregulated and 40 downregulated) differentially expressed miRNAs were selected at the three respective time points (data not shown).

GO and KEGG pathway enrichment analysis of DEGs. The results of data analysis suggested that GO terms analyzed for MSCs included biological processes (such as the positive regulation of integrin, microtubule-based process and protein polymerization), molecular functions (such as cytoskeletal protein binding and phospholipase inhibitor activity) and cellular components (such as the cell cortex, protein complex and microtubule) as presented in Fig. 1.KEGG pathway analysis for MSC differentiation-related DEGs revealed enrichment in glutathione metabolism, pathogenic Escherichia coli infection and Parkinson's disease (Fig. 2).

miRNA-mRNA interaction network. The miRNA might negatively regulate mRNA expression via degradation and translation processes. Therefore, to further investigate interaction correlations between the screened DEGs and the differentially expressed miRNAs, a miRNA-mRNA interaction network was constructed. Results from the present study demonstrated that during osteoblastic differentiation, one target gene may be regulated by several miRNAs (Fig. 3). This was also observed in adipocytes (Fig. 4). For example, upregulated DEGs, including neuronal growth regulator 1 (NEGR1), interleukin 6 signal transducer (IL6ST), platelet-derived growth factor receptor (PDGFR), enhanced homolog (ENAH), and activated leukocyte cell adhesion molecule (ALCAM), may be targeted by $>1$ differentially expressed miRNA (Fig. 3). During adipocytic differentiation, the downregulated miRNAs, including miR-495, miR-543, miR-376a and miR-140-5p, and the upregulated miR-106a, had more target genes compared with the other miRNAs examined in adipocytic differentiation (Fig. 4). It was also demonstrated that miR-145 was downregulated in both adipocytes and osteoblasts, and miR-140-5p was associated with the expression of CADM3 in the two differentiation pathways (Fig. 4).

\section{Discussion}

Previous studies have demonstrated that MSCs have the ability to differentiate into multiple cell types (21), and have identified possible applications of MSCs in cell replacement therapies 


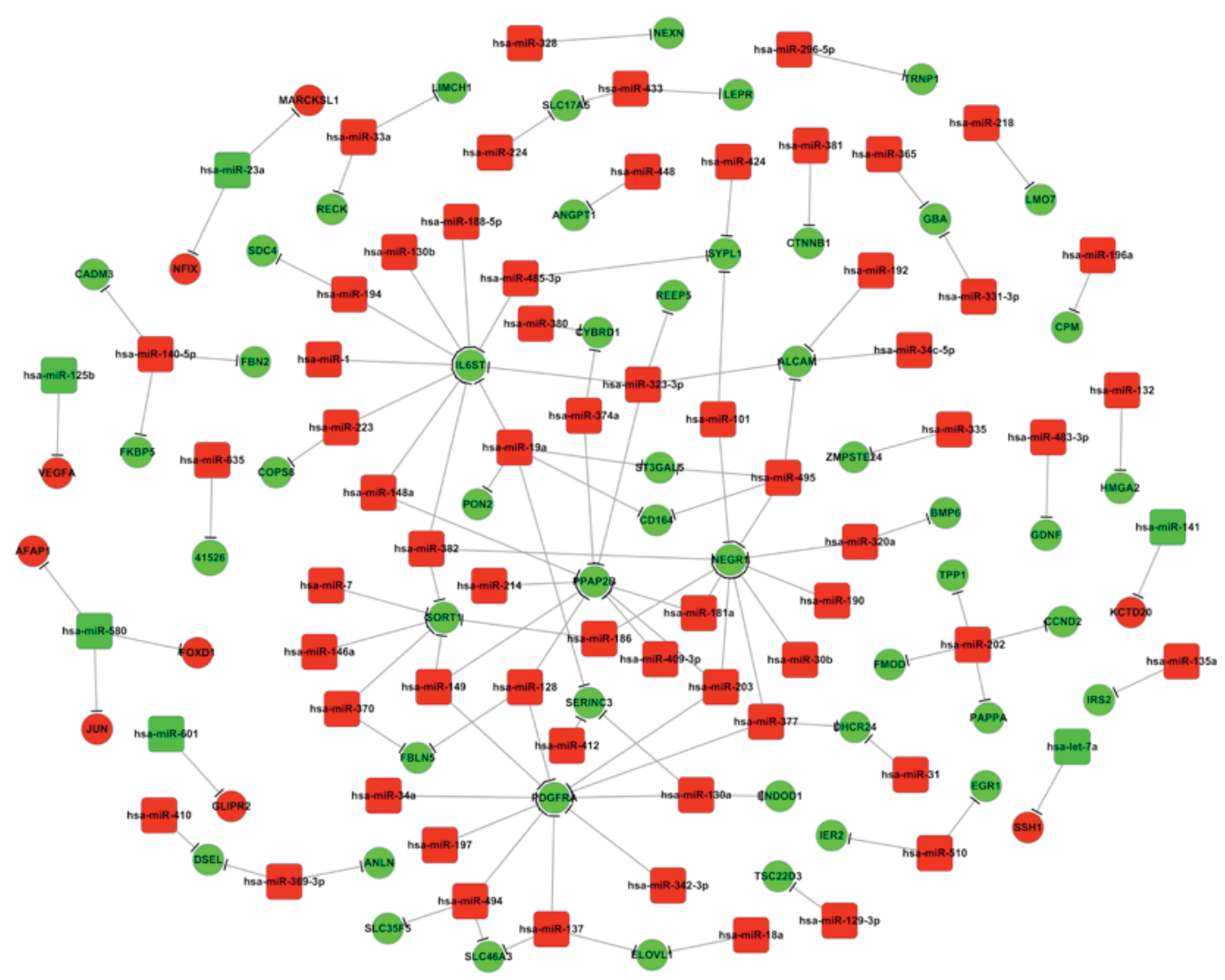

Figure 3. Interaction network for DEGs and differentially expressed miRNAs during osteoblastic differentiation of mesenchymal stem cells. Red circles indicate downregulated DEGs, green circles indicate upregulated DEGs. Red boxes indicate downregulated differentially expressed miRNAs, green boxes indicate upregulated differentially expressed miRNAs. DEGs, differentially expressed genes; miRNAs, microRNAs.

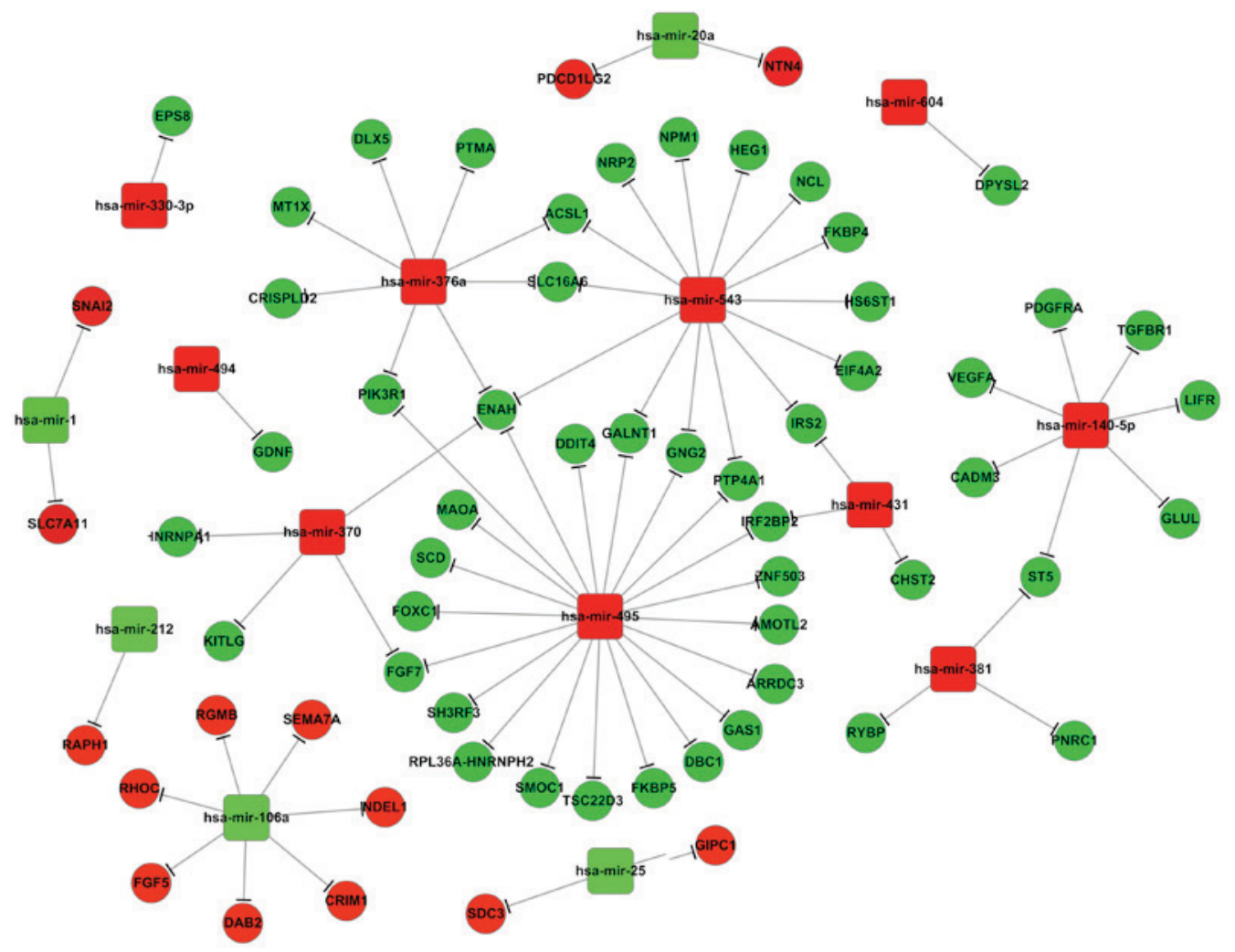

Figure 4. Interaction network for the DEGs and differentially expressed miRNAs during adipocytic differentiation of mesenchymal stem cells. Red circles indicate downregulated DEGs, green circles indicate upregulated DEGs. Red boxes indicate downregulated differentially expressed miRNAs, green boxes indicate upregulated differentially expressed miRNAs. DEGs, differentially expressed genes; miRNAs, microRNAs. 
in various diseases $(4,22)$. In the present study, DEGs and differentially expressed miRNAs were screened during MSC differentiation into either adipocytes or osteoblasts. miRNAs including miR-382 and miR-203, and DEGs including NEGR1, phosphatidic acid phosphatase 2B (PPAP2B), PDGFRA, IL6ST and sortilin 1 (SORT1) were identified as having a potential involvement in osteoblastic differentiation. In addition, the downregulated miR-495, miR-376a and miR-543, the upregulated miR-106a, the upregulated DEGs including ENAH, $N$-acetylgalactosaminyltransferase 1 (GALNT1) and acyl-CoA synthetase long-chain family member 1 (ACSL1), and the downregulated repulsive guidance molecule family member B (RGMB) and semaphorin 7A (SEMA7A) were identified as having a possible role in adipocytic differentiation.

Results from the present study suggested that miR-203 was a common regulator for certain DEGs, including NEGR1, PPAP2B and PDGFRA, whereas miR-382 was a common regulator of IL6ST, NEGR1 and SORT1 (Fig. 3), suggesting their crucial roles in regulating osteoblastic differentiation of MSCs. NEGR1 has been indicated to be involved in the cell adhesion pathway (23). Arai et al (24) demonstrated that MSCs expressed ALCAM when involved in bone marrow formation. PPAP2B, which is a member of the phosphatidic acid phosphatase family, has been revealed to convert phosphatidic acid to diacylglycerol (25); and a previous study demonstrated that PPAP2B was involved in peroxisome proliferator-activated receptor- $\gamma$-induced osteoblastic cell differentiation in MSCs (26). Alison and Islam (27) revealed that miR-203 was involved in MSC differentiation by targeting several genes. Therefore, the present study hypothesized that the downregulated expression of miR-203 may promote MSC osteoblastic differentiation, which may be associated with the upregulated expression of DEGs, including NEGR1, PPAP2B and PDGFRA. In addition, IL6ST and NEGR1 have been demonstrated to be associated with MSC osteoblastic differentiation $(26,28)$, whereas SORT1 was indicated to participate in the regulation of osteoblastic differentiation of human MSCs in vitro (29). The decay of miR-382 has been demonstrated to be important in osteoblastic differentiation of human MSCs $(10,30)$. The present study indicated that miR-382 was likely to be involved in regulating osteoblastic differentiation from human MSCs by targeting DEGs, such as IL6ST, NEGR1 and SORT1.

Results from the present study indicated that ENAH mRNA may be a common target of miRNAs, including miR-495, miR-376a, miR-543 and miR-370, suggesting a role for this DEG in MSCs differentiation into adipocytes (Fig. 4). ENAH is an actin-associated protein that is involved in various cytoskeleton remodeling and cell polarity processes, such as axon guidance and lamellipodia formation during cell migration (31). Microarray analyses by Charoenpanich et al (32) demonstrated that ENAH was differentially expressed in human adipose-derived adult stem cells undergoing osteoblastic differentiation under uniaxial cyclic tensile strain. The differentially expressed miRNAs identified in the present study, including miR-495, miR-376a, miR-543 and miR-370, have been examined in previous MSC differentiation studies. For example, Gao et al (33) revealed that miR-495 was overexpressed during MSC differentiation into osteoblasts, whereas Brodie and Slavin (34) demonstrated that downregulation of
miR-495 expression was able to be used in the astrocytogenesis induction. The present study revealed that miR-495 expression was decreased during differentiation into adipocytes. Together, these results indicated that the expression of one miRNA may be affected by different conditions or different cell types, resulting in different expression levels.

miR-543 has been demonstrated to serve a role in human adult stem cell regeneration (35). Data from the present study led to the hypothesis that the upregulated expression of ENAH may be regulated by miR-495, miR376a, miR-543 and miR-370, and thus may serve a contributing role in MSC differentiation into adipocytes.

Notably, the present study demonstrated that the expression of miR-495 was downregulated in MSCs undergoing either osteoblastic or adipocytic differentiation; however, the target DEGs of miR-495 were different (Figs. 3 and 4). For example, during osteoblastic differentiation, ALCAM, NEGR1, CD164 and ST3 $\beta$-galactoside $\alpha$-2,3-sialyltransferase 5 (ST3GAL5) were upregulated, suggesting that they may be targets for miR-495. However, none of these DEGs were identified as targets of miR-495 during MSC adipocytic differentiation. These suggested that the regulatory mechanism of the two types of MSC differentiation was different.

In conclusion, the present study screened several DEGs and differentially expressed miRNAs during osteoblastic and adipocytic differentiation of MSCs. The results revealed that DEGs, such as NEGR1, PPAP2B, PDGFRA, SORT1 and IL6ST, and differentially expressed miRNAs, including miR-203 and miR-382, may be involved in the MSCs differentiation into osteoblasts, whereas the downregulated expression of miR-495, miR-376a, miR-543 and miR-370, may be involved in regulating the expression of ENAH mRNA in MSCs differentiating into adipocytes The present study may provide a theoretical basis for further investigation into the differing pathways of MSC differentiation into osteoblasts or adipocytes, which may lead to the use of MSCs in the treatment of various diseases. Further experimental studies are required to confirm the roles of the selected DEGs or miRNAs in MSC differentiation.

\section{Acknowledgements}

The present study was supported by the National Natural Science Foundation of China (grant no. 81160113) and the Landed Science Project of Jiangxi Province Institution of Higher Education (grant no. KJLD13094).

\section{References}

1. Cells MS: Mesenchymal stem cells. Springer Berlin, 2010.

2. Wang T: Bone marrow mesenchymal stem cells and cardiovascular diseases. Chinese Journal of Critical Care Medicine, 2008.

3. Sekiya I, Muneta T, Morio T, Shimizu N and Kuroiwa Y: Application of synovium-derived mesenchymal stem cells (mscs) for cartilage or meniscus regeneration. Va Us, 2008.

4. Barry FP and Murphy JM: Mesenchymal stem cells: Clinical applications and biological characterization. Int J Biochem Cell Biol 36: 568-584, 2004.

5. Hamidouche Z, Fromigué O, Nuber U, Vaudin P, Pages JC, Ebert R, Jakob F, Miraoui H and Marie PJ: Autocrine fibroblast growth factor 18 mediates dexamethasone-induced osteogenic differentiation of murine mesenchymal stem cells. J Cell Physiol 224: 509-515, 2010. 
6. Sammons J, Ahmed N, El-Sheemy M and Hassan HT: The role of BMP-6, IL-6 and BMP-4 in mesenchymal stem cell-dependent bone development: Effects on osteoblastic differentiation induced by parathyroid hormone and vitamin $\mathrm{D}(3)$. Stem Cells Dev 13: 273-280, 2004.

7. Jing L, Jia Y, Lu J, Han R, Li J, Wang S, Peng T and Jia Y: MicroRNA-9 promotes differentiation of mouse bone mesenchymal stem cells into neurons by Notch signaling. Neuroreport 22: 206-211, 2011

8. Vam Pham P, Hong-Thien Bui K, Quoc Ngo D, Tan Khuat L and Kim Phan N: Transplantation of nonexpanded adipose stromal vascular fraction and platelet-rich plasma for articular cartilage injury treatment in mice model. J Med Eng 2013: 832396, 2013

9. Wan Q, Guan SJ, LI XC, Sun CP, Zhou JX, Xia B, Xia TT and Zeng QS: Imaging features of thoracic well-differentiated and myxoid liposarcoma: Comparison with pathology. Chin J Med Imag Technol 30: 78-81, 2014.

10. Arfat Y, Xiao WZ, Ahmad M, Zhao F, Li DJ, Sun YL, Hu L, Zhihao C, Zhang G, Iftikhar S, et al: Role of microRNAs in osteoblasts differentiation and bone disorders. Curr Med Chem 22: 748-758, 2015.

11. Longhi A, Neri S, Speranza C, Alberghini M, Ferrari C, Abate M, Cesari M, Ferrari S, Palmerini E and Mercuri M Liposarcoma treatment: Role of radiotherapy and chemotherapy. J Clin Oncol 26: 431-436, 2008.

12. Mithoefer K, McAdams TR, Scopp JM and Mandelbaum BR Emerging options for treatment of articular cartilage injury in the athlete. Clin Sports Med 28: 25-40, 2009

13. Chen TS, Lai RC, Lee MM, Choo AB, Lee CN and Lim SK: Mesenchymal stem cell secretes microparticles enriched in pre-microRNAs. Nucleic Acids Res 38: 215-224, 2010.

14. Luca AD: RNA-seq analysis reveals significant effects of EGFR signalling on the secretome of mesenchymal stem cells. Oncotarget 5: 10518-10528, 2014.

15. Mrugala D, Dossat N, Ringe J, Delorme B, Coffy A, Bony C, Charbord P, Häupl T, Daures JP, Noël D and Jorgensen C: Gene expression profile of multipotent mesenchymal stromal cells: Identification of pathways common to TGF beta 3/BMP2-induced chondrogenesis. Cloning Stem Cells 11: 61-76, 2009.

16. Trapnell C, Pachter L and Salzberg SL: TopHat: Discovering splice junctions with RNA-Seq. Bioinformatics 25: 1105-1111, 2009.

17. Harris MA, Clark J, Ireland A, Lomax J, Ashburner M, Foulger R, Eilbeck K, Lewis S, Marshall B, Mungall C, et al: The gene ontology (GO) database and informatics resource. Nucleic Acids Res 32 (Database Issue): D258-D261, 2004.

18. Altermann E and Klaenhammer TR: PathwayVoyager: Pathway mapping using the Kyoto encyclopedia of genes and genomes (KEGG) database. BMC Genomics 6: 60, 2005.

19. Dennis G Jr, Sherman BT, Hosack DA, Yang J, Gao W, Lane HC and Lempicki RA: DAVID: Database for annotation, visualization and integrated discovery. Genome Biol 4: P3, 2003.

20. Varambally S, Cao Q, Mani RS, Shankar S, Wang X, Ateeq B, Laxman B, Cao X, Jing X, Ramnarayanan K, et al: Genomic loss of microRNA-101 leads to overexpression of histone methyltransferase EZH2 in cancer. Science 322: 1695-1699, 2008.
21. Beyer Nardi N and da Silva Meirelles L: Mesenchymal stem cells: Isolation, in vitro expansion and characterization. Handb Exp Pharmacol 249-282, 2006.

22. Schiavi-Tritz J, Reppel L, Magdalou J, Stoltz JF and Huselstein C: 229 improving hBM-MSCs differentiation DM a stratified scaffold for osteoarticular repairing. Osteoarthrit Cartil S112, 2011.

23. Funatsu N, Miyata S, Kumanogoh H, Shigeta M, Hamada K, Endo Y, Sokawa Y and Maekawa S: Characterization of a novel rat brain glycosylphosphatidylinositol-anchored protein (Kilon), a member of the IgLON cell adhesion molecule family. J Biol Chem 274: 8224-8230, 1999.

24. Arai F, Ohneda O, Miyamoto T, Zhang XQ and Suda T: Mesenchymal stem cells in perichondrium express activated leukocyte cell adhesion molecule and participate in bone marrow formation. J Exp Med 195: 1549-1563, 2002.

25. Sánchez-Sánchez R, Morales-Lázaro SL, Baizabal JM, Sunkara M, Morris AJ and Escalante-Alcalde D: Lack of lipid phosphate phosphatase-3 in embryonic stem cells compromises neuronal differentiation and neurite outgrowth. Dev Dyn 241: 953-964, 2012.

26. Shockley KR, Lazarenko OP, Czernik PJ, Rosen CJ, Churchill GA and Lecka-Czernik B: PPARgamma2 nuclear receptor controls multiple regulatory pathways of osteoblast differentiation from marrow mesenchymal stem cells. J Cell Biochem 106: 232-246, 2009.

27. Alison MR and Islam S: Attributes of adult stem cells. J Pathol 217: 144-160, 2009.

28. Shockley KR, Rosen CJ, Churchill GA and Lecka-Czernik B: PPARgamma2 regulates a molecular signature of marrow mesenchymal stem cells. PPAR Res 2007: 81219, 2007.

29. Kulterer B, Friedl G, Jandrositz A, Sanchez-Cabo F, Prokesch A, Paar C, Scheideler M, Windhager R, Preisegger KH and Trajanoski Z: Gene expression profiling of human mesenchymal stem cells derived from bone marrow during expansion and osteoblast differentiation. BMC Genomics 8: 70, 2007.

30. Kapinas K and Delany AM: MicroRNA biogenesis and regulation of bone remodeling. Arthritis Res Ther 13: 220, 2011.

31. Gurzu S, Ciortea D, Ember I and Jung I: The possible role of mena protein and its splicing-derived variants in embryogenesis, carcinogenesis, and tumor invasion: A systematic review of the literature. Biomed Res Int 2013: 365192, 2013.

32. Charoenpanich A, Wall ME, Tucker CJ, Andrews DM, Lalush DS and Loboa EG: Microarray analysis of human adipose-derived stem cells in three-dimensional collagen culture: Osteogenesis inhibits bone morphogenic protein and Wnt signaling pathways, and cyclic tensile strain causes upregulation of proinflammatory cytokine regulators and angiogenic factors. Tissue Eng Part A 17: 2615-2627, 2011.

33. Gao J, Yang T, Han J, Yan K, Qiu X, Zhou Y, Fan Q and Ma B: MicroRNA expression during osteogenic differentiation of human multipotent mesenchymal stromal cells from bone marrow. J Cell Biochem 112: 1844-1856, 2011.

34. Brodie C and Slavin S: MicroRNAs for the generation of astrocytes. WO, 2014

35. Ng TK, Carballosa CM, Pelaez D, Wong HK, Choy KW, Pang CP and Cheung HS: Nicotine alters MicroRNA expression and hinders human adult stem cell regenerative potential. Stem Cells Dev 22: 781-790, 2013. 